\title{
Estimativa da produtividade de café conilon utilizando técnicas de cokrigagem
}

\author{
Julião Soares de Souza Lima ${ }^{*}$, Samuel de Assis Silva ${ }^{2}$, Rone Batista de Oliveira ${ }^{3}$, Abel Souza da Fonseca
}

\author{
$10.1590 / 0034-737 X 201663010008$
}

\begin{abstract}
RESUMO
Estimar a variabilidade espacial de uma cultura em campo auxilia no entendimento de alguns fenômenos que podem estar correlacionados com a sua produtividade. Este trabalho teve, como objetivo, estimar, pelo método da cokrigagem, a produtividade $\left(\mathrm{kg} \mathrm{ha}^{-1}\right)$ do cafeeiro conilon, em três safras consecutivas, tendo como covariável o número de ramos produtivos (plagiotrópicos) por planta. No centro de uma lavoura, demarcou-se uma malha amostral, com 109 pontos georreferenciados, sendo cada ponto constituído por cinco plantas. Fez-se o acompanhamento, por três safras agrícolas consecutivas, contando-se os ramos produtivos e realizando-se a colheita manual. O café colhido foi separado, para cada estádio de maturação (cereja, verdoengo e verde), e secado para umidade padrão de 12\%, determinando-se a produtividade de café seco em coco $\left(\mathrm{kg} \mathrm{ha}^{-1}\right)$. A produtividade e o número de ramos produtivos por ponto amostral apresentaram correlação linear e dependência espacial nas três safras. As estimativas das produtividades pelos números de ramos produtivos apresentaram similaridades na cokrigagem, mostrando ser a covariável ramo produtivo eficiente na estimativa da produtividade do café conilon (Robusta Tropical).
\end{abstract}

Palavras-chave: geoestatística, variabilidade espacial, variograma cruzado.

\section{ABSTRACT}

\section{Use of kriging techniques to estimate Conilon coffee productivity}

Knowing the spatial variability of a coffee-crop helps to understand some phenomena which can be correlated with its productivity. This study aimed estimating the productivity $\left(\mathrm{kg} \mathrm{ha}^{-1}\right)$ of conilon coffee-tree in three consecutive harvests using the cokriging method, considering the number of plagiotropic branches per plant as a covariable. In the center of a coffee-crop we settled a sampling grid with 109 georeferenced points, each point consisted of five plants. Subsequently the vegetative branches were counted for three consecutive growing seasons before performing manual harvesting of coffee beans. The harvested coffee was separated into cherry, yellowish and green and dried to a standard moisture of $12 \%$. The dry cherry productivity $\left(\mathrm{kg} \mathrm{ha}^{-1}\right)$ was then evaluated. The three harvests evaluated showed linear correlation and spatial dependence among productivity and number of vegetative branches. Estimation of productivity by the number of vegetative branches showed similarities in cokriging, indicating that vegetative branches is an efficient covariable to estimate conilon coffee productivity.

Key words: geostatistics, spatial variability, cross semivariogram.

\footnotetext{
Submetido em 06/02/2013 e aprovado em 08/10/2015.

${ }^{1}$ Universidade Federal do Espírito Santo, Centro de Ciências Agrárias, Departamento de Engenharia Rural, Alegre, Espírito Santo, Brasil. limajss@yahoo.com.br

${ }^{2}$ Universidade Federal do Espírito Santo, Centro de Ciências Agrárias, Departamento de Engenharia Rural, Alegre, Espírito Santo, Brasil. sasilva@pq.cnpq.br

${ }^{3}$ Universidade Estadual do Norte do Paraná, Centro de Ciências Agrárias, Departamento de Engenharia Rural, Bandeirantes, Paraná, Brasil. ronebatista@hotmail.com

${ }^{4}$ Universidade Federal do Espírito Santo, Centro de Ciências Agrárias, Departamento de Engenharia Rural, Alegre, Espírito Santo, Brasil. abelufes@hotmail.com

*Autor para correspondência: limajss@yahoo.com.br
} 


\section{INTRODUÇÃO}

Pesquisas têm sido realizadas com o cafeeiro conilon, no intuito de determinar a dependência espacial de variáveis do solo e as relativas às plantas, em separado ou correlacionadas, na tentativa de interpretar e otimizar a aplicação de insumos. A utilização conjunta de diferentes variáveis do sistema solo-planta pode conduzir a resultados mais confiáveis, no levantamento de dados, oferecendo informações mais precisas para o gerenciamento do agronegócio cafeeiro (Silva et al., 2014), identificando e interpretando, por exemplo, zonas de diferentes produtividades.

Os métodos geoestatísticos para interpretar a variabilidade espacial da fertilidade do solo do cafeeiro conilon permitem reduzir o número de variáveis e, também, a construção de mapas de classes pelo método de interpolação krigagem ordinária, facilitando a identificação da presença de zonas de diferentes manejos na área (Lima et al., 2009).

Em vários estudos de análise de dependência espacial em variáveis de solo, tem-se utilizado o método da cokrigagem, que descreve as variações espacial ou temporal simultâneas de duas variáveis aleatórias que têm forte associação entre si (Silva et al., 2010a). Bottega et al. (2011) estimaram por cokrigagem os teores de Ca e $\mathrm{Mg}$, utilizando, como covariável, o pH do solo, o que se mostrou eficiente nas estimativas.

Silva et al. (2010b) estimaram o K e o Mg do solo, tendo como covariáveis as frações granulométricas areia total e silte, respectivamente. Verificaram que a utilização da areia total superestima valores do K, na região mais alta da área, e os subestima, nas regiões mais baixas. A estimativa de $\mathrm{Mg}$ em função do silte possibilitou obter um mapa representativo da sua distribuição com menor erro.

No que diz respeito à produtividade do cafeeiro conilon, Oliveira (2007) afirma que bons resultados para esta característica passam pelo entendimento da distribuição espacial do número de ramos produtivos. Segundo Ferrão et al. (2008), o número ideal de ramos produtivos ainda é uma busca dos estudos sobre o manejo de podas nos cafeeiros conilon, visto que estão relacionados com aspectos fisiológicos e nutritivos da planta e determinam as cargas de frutos.

Oliveira (2007), estudando a distribuição espacial de ramos produtivos em duas safras consecutivas, observou dependência espacial na primeira safra e ausência na segunda. Segundo o autor, pode-se dizer que os fenômenos que regeram o número de ramos produtivos nas safras não foram semelhantes, sendo provável que o efeito climático, o tipo de poda e a mão de obra ao longo dos anos tenham sido os maiores responsáveis por estas diferenças, uma vez que cada planta do cafeeiro conilon apresenta arquitetura diferente.
Este trabalho tem como objetivo estimar a produtividade do cafeeiro conilon em três safras consecutivas, por meio do método de cokrigagem, utilizando-se como covariável o número de ramos produtivos (plagiotrópicos) por planta.

\section{MATERIAL E MÉTODOS}

$\mathrm{O}$ experimento foi realizado na área do Incaper, no município de Cachoeiro de Itapemirim, ES, cultivada com café conilon (Robusta Tropical, Emcaper 8151), nas coordenadas $20^{\circ} 45^{\prime} 17.31^{\prime \prime} \mathrm{S}$ e de $41^{\circ} 17^{\prime} 8.86^{\prime \prime} \mathrm{O}$. A área está situada no terço superior de uma elevação com topo arredondado e vertente perfil convexo, altitude média de 113 $\mathrm{m}$ e declividade de $16 \%$. O solo foi classificado como Latossolo Vermelho-Amarelo distrófico com textura argilosa (Oliveira, 2007). Na profundidade de 0,0-0,20 m, as frações granulométricas médias são: argila $=414,7$; silte $=$ 190,5; areia total $=393,9\left(\mathrm{~g} \mathrm{~kg}^{-1}\right)$.

Os pontos de amostragens foram georreferenciados com GPS topográfico, totalizando-se 109 pontos amostrais, sendo cada ponto composto de cinco plantas, perfazendo-se área por ponto de $13,05 \mathrm{~m}^{2}$, no espaçamento de 2,5 $\mathrm{m}$ entre linhas e de $0,90 \mathrm{~m}$ entre plantas, na linha, totalizando-se 3831 plantas ha-1.

O trabalho foi conduzido em três safras consecutivas (01, 02 e 03), nos anos de 2005, 2006 e 2007. A contagem dos ramos plagiotrópicos produtivos (RP) foi realizada, sempre, aos sete dias antes das colheitas, por derriça manual (mês de junho), de todos os ramos produtivos (plagiotrópicos) por planta, com café nos estádios cereja, verdoengo e verde, por ponto amostral. Após cada colheita, seguiram-se os critérios adotados na lavoura para a realização das práticas de poda (retirada dos ramos ortotrópicos e plagiotrópicos não produtivos) e de correção da fertilidade do solo.

Considerando-se que a precipitação pluvial mensal (mm) é um fator ambiental que influencia o desenvolvimento da cultura do cafeeiro, principalmente na época da emissão dos botões florais, apresentam-se , a seguir, os meses com as suas respectivas precipitações (mm), para auxiliar na análise dos dados: a) primeira safra: maio $=126$, junho $=68$, julho $=33$, agosto $=45$ e setembro $=61 ; b$ ) segunda safra: maio $=126$, junho $=68$, julho $=33$, agosto 22 e setembro $=101$; c) terceira safra: maio $=14$, junho $=$ 13 , julho $=4$, agosto $=10$ e setembro $=78$.

O café colhido foi transportado, em sacos etiquetados, para o laboratório do Instituto de Pesquisa, Assistência Técnica e Extensão Rural - Incaper, para a pesagem e retirada de amostras separadas por estádios de maturação do café, conforme descrito por Oliveira (2007), a fim de corrigir a produtividade para a umidade padrão de $12 \%$, obtendo-se a produtividade do café seco em coco. 
Após análise dos dados em laboratório, fez-se a análise exploratória para verificar a presença de dados discrepantes (outliers), considerando-se os quartis superiores e inferiores, definidos pelo box-plot, e observando-se sua influência nas medidas de posição e de dispersão.

Foi realizada análise descritiva, para determinação das médias de posição e de dispersão dos dados, e a normalidade foi verificada pelo teste Kolmogorov-Smirnov (valor-p < 0,05). Análise qualitativa foi realizada pelo teste de Tukey (valor-p < 0,05) entre as variáveis nas diferentes safras. Foi realizada análise de correlação linear de Pearson (valor-p < 0,05), para determinação da relação entre as variáveis das plantas (produtividade e ramos produtivos), considerando-se a classificação de Kitamura et al. (2007).

As análises geoestatísticas univariada e multivariada foram realizadas, considerando-se a hipótese de estacionaridade assumida (Journel \& Huibregts, 1978) por meio de ajuste do semivariograma, estimado pela seguinte equação:

$\gamma(\boldsymbol{h})=\frac{1}{2 N(\boldsymbol{h})} \sum_{i=1}^{N(\boldsymbol{h})}\left[Z_{1}\left(x_{i}\right)-Z_{2}\left(x_{i}+\boldsymbol{h}\right)\right]^{2}$

em que: $N(\boldsymbol{h})$ é o número de pares de valores $\left[Z\left(x_{i}\right), Z\left(x_{i}+\right.\right.$ $\boldsymbol{h})$ ] separados por um vetor $\mathbf{h}$ e, $x_{i} a$ posição espacial da variável $Z$.

À nuvem de pontos $[h, \gamma(\boldsymbol{h})]$ ajusta-se uma função matemática, cujos parâmetros são conhecidos como: efeito pepita $\left(\mathrm{C}_{0}\right)$, correspondendo ao valor da interseção no eixo das semivariâncias; patamar $\left(\mathrm{C}_{0}+\mathrm{C}\right)$, aproximadamente igual ao valor da variância dos dados; e alcance (a), que representa a distância na qual o semivariograma atinge o valor do patamar e define a região de dependência espacial entre as amostras. Os parâmetros $\left(\mathrm{C}_{0}\right.$ e $\left.\mathrm{C}_{0}+\mathrm{C}\right)$ dos semivariogramas foram escalonados pela variância e, o dos semivariogramas cruzados, pela covariância dos dados, para efeito de padronização de escala.

A escolha do modelo adotado baseou-se na minimização da soma dos quadrados dos resíduos (SQR) e no coeficiente de determinação múltipla $\left(\mathrm{R}^{2}\right)$, do ajuste dos modelos teóricos aos semivariogramas experimentais. $\mathrm{Na}$ sequência, também foi utilizado o coeficiente de correlação da validação cruzada (r-VC) (valores observados versus valores estimados), como critério de escolha (Lima et al., 2007).

Para análise do grau de dependência espacial (GDE\%), foram utilizados os intervalos definidos por Cambardella et al. (1994), na relação $\left[\mathrm{C}_{0} /\left(\mathrm{C}_{0}+\mathrm{C}\right)\right]^{*} 100$, que considera o grau de dependência espacial forte $(\mathrm{GDE} \leq 25 \%)$; mode$\operatorname{rado}(25 \%<\mathrm{GDE} \leq 75 \%)$ e baixo $(\mathrm{GDE}>75 \%)$.

Para estimar valores de ramos produtivos (RP) e da produtividade de café $\left(\mathrm{kg} \mathrm{ha}^{-1}\right)$, nas três safras (Prod 01, 02 e 03 ), em locais não amostrados, e confeccionar os mapas da distribuição espacial, utilizou-se o método de interpolação krigagem ordinária. Este interpolador geoestatístico utiliza um estimador linear não viciado, com mínima variância, e leva em consideração a estrutura de variabilidade espacial encontrada para cada variável (Isaaks \& Srivastara, 1989).

Para estimar a produtividade $\left(\mathrm{kg} \mathrm{ha}^{-1}\right)$ em função dos ramos produtivos (RP), em cada safra, utilizou-se a extensão multivariada da krigagem, conhecida como cokrigagem, como demostrado por Isaaks \& Srivastava, (1989) e pelo estudo de Silva et al., (2010a). Este método baseia-se nos parâmetros expressos por um semivariograma cruzado entre duas variáveis, para a estimativa de novos valores em locais não amostrados, conforme a equação.

$$
\begin{aligned}
& \gamma_{12}(\boldsymbol{h})=\frac{1}{2 N(\boldsymbol{h})}\left\{\sum _ { i = 1 } ^ { N ( \boldsymbol { h } ) } [ Z _ { 1 } ( x _ { i } + \boldsymbol { h } ) - Z _ { 1 } ( x _ { i } ) ] * \left[Z_{2}\left(x_{i}+\boldsymbol{h}\right)-.\right.\right. \\
& \left.\left.-Z_{2}\left(x_{i}\right)\right]\right\}
\end{aligned}
$$

O método cokrigagem é utilizado quando amostras são coletadas, de preferência na sua totalidade, dentro de um mesmo domínio espacial, e apresentam significativo grau de correlação (Goovaerts, 1997; Landim et al., 2002; Soares, 2006). A estimativa de uma variável $Z^{*}\left(x_{0}\right)$ para qualquer local $\mathrm{x}_{0}$ deve ser uma combinação linear de $Z_{1} \mathrm{e}$ $Z_{2}$, ou seja:

$Z^{*}\left(x_{0}\right)=\sum_{i=1}^{n_{2}} \lambda_{1 i} Z_{1}\left(x_{1 i}\right)+\sum_{j=1}^{n_{2}} \lambda_{2 j} Z_{2}\left(x_{2 j}\right)$

em que: $\mathrm{n}_{1}$ e $\mathrm{n}_{2}$ são os números de vizinhos medidos de $Z_{1}$ e $Z_{2}$, respectivamente, e $\lambda_{1}$ e $\lambda_{2}$ são os ponderadores associados a $Z_{1}$ e $Z_{2}$ os quais são distribuídos de acordo com a dependência espacial de cada uma das variáveis entre si e com a correlação cruzada entre elas. Da mesma forma que na krigagem, para que este estimador seja ótimo, ele também deve ter variância mínima e ser não tendencioso (Landim et al., 2002).

\section{RESULTADOS E DISCUSSÃO}

A análise descritiva dos dados de ramos produtivos (RP) e da produtividade (Prod), para a cultura do cafeeiro conilon, em três safras consecutivas, está apresentada na Tabela 1, sem a presença de dois pontos discrepantes na produtividade, na primeira e na segunda safras, respectivamente.

Os ramos produtivos (RP) apresentaram valores médios significativamente diferentes pelo teste de Tukey (valor-p < 0,05), entre as diferentes safras. No entanto, as produtividades nas safras 01 (Prod01) e 02 (Prod02) não diferiram significativamente entre si, sendo que ambas apresentaram diferenças significativas com a da safra 03 (Prod03). 
Os ramos produtivos, em cada safra, são produtos da prática de poda que se realiza após cada colheita, que consiste na retirada dos ramos (ortotrópicos e plagiotrópicos), com produção esgotada em função dos anos de produção. Segundo Ferrão et al. (2007), a manutenção desses ramos na lavoura pode, também, contribuir para a obstrução da passagem da luz solar para o interior da planta, refletindo-se na diminuição da produção. Mas, nesse caso, na safra 03, a produtividade caiu aproximadamente 2,5 vezes, em relação às duas anteriores. Esta diferença está relacionada com a precipitação pluvial mensal, quantitativamente superior e melhor distribuída nos meses de desenvolvimento do botão floral, no cafeeiro (de maio a setembro nas safras 01 e 02) e com o sistema de poda adotado na safra anterior. Segundo Berlato et al. (2005), a instabilidade climática influencia acentuadamente a variabilidade temporal da produtividade das culturas.

Todos os atributos estudados apresentaram distribuição normal, pelo teste Kolmogorov-Smirnov. Segundo Isaaks \& Srivastarava (1989), quando os dados não apresentarem distribuição normal, o importante é que a distribuição não apresente cauda muito alongada, o que pode influenciar a definição do patamar e o ajuste do semivariograma, o que não ocorreu. As produtividades, nas três safras, apresentaram distribuição com assimetria à direita (ks), com valor médio maior que a mediana (Q2) e com concentração de valores abaixo da média.
Os coeficientes de variação $(\mathrm{CV})$ para todas as variáveis apresentaram média variação $(12 \%<\mathrm{CV}<60 \%)$, com exceção para a produtividade, na safra 03 (Prod03) que apresentou valor alto de 87,0\% (CV > 60\%), conforme classificação proposta por Warrick \& Nielsen (1980).

Na Tabela 2, estão apresentadas as correlações lineares de Pearson (valor-p < 0,05) entre as variáveis estudadas (RPxProd). Observam-se altos coeficientes de correlações, nas safras 01 e 03, para o RPxProd, com r = 0,63 e r = 0,61, respectivamente, e baixa correlação, na safra 02 , com $r=$ 0,41 , entre ramos produtivos versus produtividade. A ausência de correlação significativa entre ramos produtivos (RP01xRP02) e produtividade (Prod01xProd02), nas safras 01 e 02 , é explicada pela ocorrência das maiores e menores produtividades, em regiões diferentes, entre os dois anos. A existência da correlação entre as variáveis é uma premissa fundamental para a utilização da cokrigagem.

$\mathrm{Na}$ análise espacial com ajuste dos dados aos semivariogramas simples de cada variável, verificou-se que elas apresentam dependência espacial (Tabela 3). O ajuste dos modelos permitiu construir semivariogramas com patamares bem definidos (Figura 1). Dessa forma, a hipótese intrínseca, que é a exigência mínima para a análise geoestatística, foi atendida.

O alcance (a) de dependência espacial é um parâmetro importante no estudo da variabilidade espacial, indica a zona de influência de uma amostra, ou seja, define a distância máxima até onde o valor de uma variável apresenta

Tabela 1: Análise descritiva do número de ramos produtivos de cafeeiro conilon e das produtividades, em três safras consecutivas

\begin{tabular}{|c|c|c|c|c|c|c|c|c|c|c|c|}
\hline Variável & Média & Q2 & Min. & Max. & Q1 & Q3 & $\mathbf{S}$ & Ks & kc & CV & DN \\
\hline RP01 & $3,6 b$ & 3,6 & 1,8 & 5,2 & 3,0 & 4,2 & 0,8 & $-0,19$ & $-0,81$ & 21,9 & $\mathrm{~N}$ \\
\hline Prod01 & $6636,5 \mathrm{a}$ & 6441,1 & 2701,2 & 14421,3 & 5096,6 & 7890,8 & 2189,1 & 0,55 & 0,70 & 33,0 & $\mathrm{~N}$ \\
\hline RP02 & $4,0 \mathrm{a}$ & 4,0 & 2,6 & 4,8 & 3,6 & 4,4 & 0,5 & $-0,52$ & $-0,46$ & 13,3 & $\mathrm{~N}$ \\
\hline Prod02 & $6990,1 \mathrm{a}$ & 6867,4 & 2599,8 & 13334,3 & 5393,6 & 8063,6 & 2043,5 & 0,49 & $-0,01$ & 29,2 & $\mathrm{~N}$ \\
\hline RP03 & $2,8 \mathrm{c}$ & 3,0 & 0,0 & 5,0 & 2,0 & 3,6 & 1,1 & $-0,26$ & $-0,54$ & 39,1 & $\mathrm{~N}$ \\
\hline Prod03 & $2956,8 b$ & 2219,4 & 195,1 & 13456,4 & 953,7 & 4391,9 & 2572,9 & 1,43 & 2,39 & 87,0 & $\mathrm{~N}$ \\
\hline
\end{tabular}

RP01, 02 e 03: número de ramos produtivos médios nas safras 01, 02 e 03; Prod01, 02 e 03: produtividade média de café seco em coco (kg ha $^{-1}$ ) nas três safras; Q1: primeiro quartil; Q2: mediana; Q3: terceiro quartil; S: desvio padrão; Min.: valor mínimo; Max.: valor máximo; ks; coeficiente de assimetria; kc: coeficiente de curtose; CV: coeficiente de variação; N: distribuição normal pelo teste KolmogorovSmirnov $(\mathrm{p}<0,05)$.

Médias seguidas, em coluna, por mesma letra para um mesmo atributo não diferem significativamente pelo teste de Tukey (valor-p < 0,05$)$.

Tabela 2: Correlação de Pearson ( r ) (valor-p < 0,05) entre as variáveis em estudo número de ramos produtivos (RP) e produtividade (Prod)

\begin{tabular}{lcccccc}
\hline Variável & RP01 & Prod 01 & RP02 & Prod 02 & RP03 & Prod 03 \\
\hline RP 01 & 1,00 & $\mathbf{0 , 6 3}$ & - & - & - & - \\
Prod 01 & & 1,00 & - & - & - & - \\
RP 02 & & 1,00 & $\mathbf{0 , 4 1}$ & - & $\mathbf{0 , 2 8}$ \\
Prod 02 & & & 1,00 & - & $\mathbf{0 , 6 1}$ \\
RP 03 & & & & 1,00 & 1,00 \\
Prod 03 & & & & & \\
\hline
\end{tabular}

r em negrito, significativo a $5 \%$ 
Julião Soares de Souza Lima et al.

Tabela 3: Modelos e parâmetros escalonados dos semivariogramas simples e cruzados

\begin{tabular}{lccccccc}
\hline Variável & Modelo & $\mathbf{C}_{\mathbf{0}}$ & $\mathbf{C}_{\mathbf{0}}+\mathbf{C}$ & $\mathbf{a}(\mathbf{m})$ & $\mathbf{G D E}(\boldsymbol{\%})$ & $\mathbf{R}^{\mathbf{2}}(\boldsymbol{\%})$ & $\mathbf{r}-\mathbf{V C}$ \\
\hline RP 01 & ESF & 0,59 & 1,11 & 42,0 & 40,0 & 93 & 0,48 \\
Prod 01 & ESF & 0,37 & 1,12 & 42,0 & 33,0 & 90 & 0,62 \\
RP 02 & EXP & 0,25 & 1,02 & 13,0 & 34,0 & 92 & 0,50 \\
Prod 02 & EXP & 0,02 & 1,00 & 17,0 & 2,0 & 91 & 0,42 \\
RP 03 & EXP & 0,52 & 1,06 & 50,0 & 50,0 & 93 & 0,45 \\
Prod 03 & EXP & 0,18 & 1,10 & 52,0 & 26,0 & 96 & 0,62 \\
\hline & & \multicolumn{7}{c}{ Semivariograma Simples } \\
\hline Prod01xRP01 & ESF & 0,21 & 1,00 & 45,0 & 29,0 & 92,0 & 0,55 \\
Prod02xRP02 & ESF & 0,43 & 1,00 & 38,0 & 48,0 & 70,0 & 0,51 \\
Prod03xRP03 & EXP & 0,14 & 1,00 & 66,0 & 28,0 & 99,0 & 0,62 \\
\hline C:
\end{tabular}

$\mathrm{C}_{0}$ : efeito pepita; $\mathrm{C}_{0}+\mathrm{C}$ : patamar; a: alcance de dependência espacial; GDE: grau de dependência espacial; $\mathrm{R}^{2}$ : coeficiente de determinação múltipla; r-VC: coeficiente de correlação linear entre os valores observados e os estimados na escolha do modelo; ESF: modelo esférico; EXP: modelo exponencial; Prod01, 02 e 03x RP01, 02 e 03: ajuste das variáveis no semivariograma cruzado.

relação de dependência espacial com o seu vizinho (Silva et al., 2010c). As variáveis RP01 e Prod01 apresentaram ajustes ao modelo esférico, com alcances de $42,0 \mathrm{~m}$, respectivamente. Na safra 02 , o alcance para RP02 foi de 13,0 m e para Prod02 de 17,0 m, com ajustes ao modelo exponencial. Na safra 03, o RP e a Prod ajustaram-se ao modelo exponencial, com alcances de $50 \mathrm{~m}$ e 52, respectivamente. De acordo com McBratney e Webster (1986), os
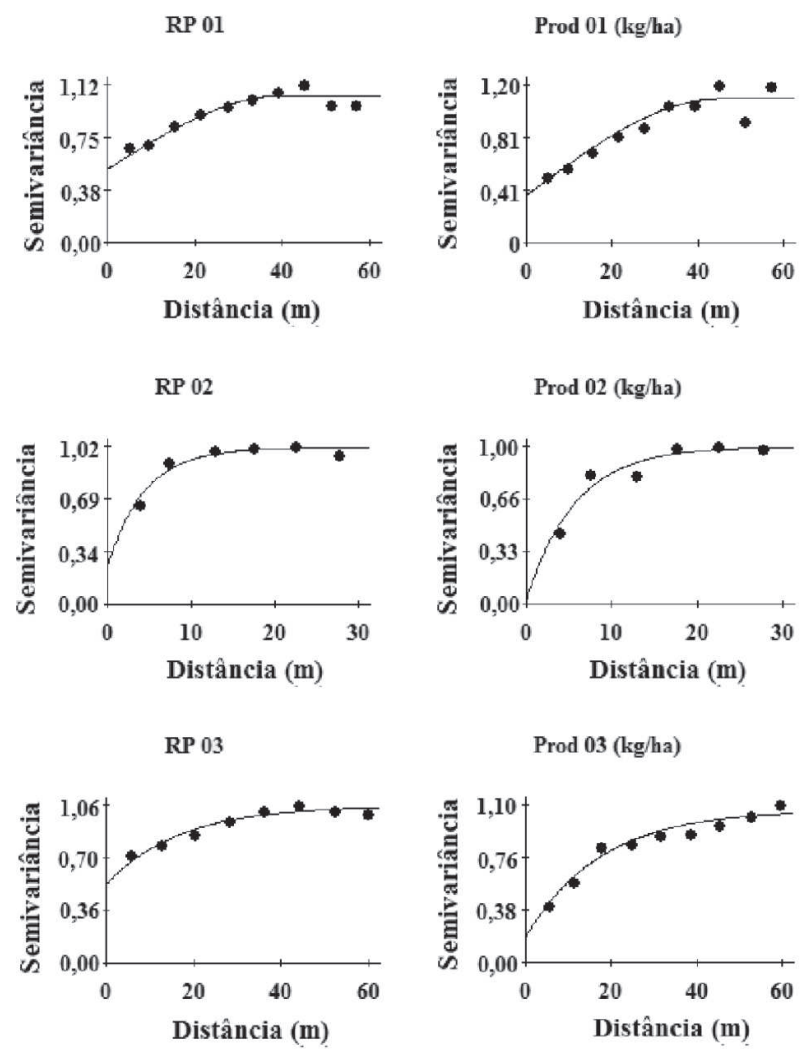

Figura 1: Semivariogramas escalonadas dos atributos Ramos Produtivos (RP) e Produtividade (Prod), para três safras consecutivas do cafeeiro conilon.

Rev. Ceres, Viçosa, v. 63, n.1, p. 054-061, jan-fev, 2016 modelos matemáticos que mais se ajustam às variáveis do solo são o esférico (ESF) e o exponencial (EXP).

A análise de dependência espacial confirma que ramos produtivos e produtividade, para uma mesma safra, apresentam correlações espaciais. Sabe-se que as plantas do cafeeiro conilon têm arquitetura diferente daquela do cafeeiro arábica, com mais de um ramo plagiotrópico produzindo, normalmente. Com estes resultados, a variável ramo produtivo pode ser utilizada na predição da produtividade, além de fácil de ser quantificada em campo.

Para os ajustes dos semivariogramas simples, as variáveis apresentaram fortes graus de dependência para a Prod02 (GDE $\leq 25 \%)$, mostrando as demais variáveis dependências moderada $(25 \%<\mathrm{GDE}<75 \%)$, valores estes influenciados pelo valor do efeito pepita $\left(\mathrm{C}_{0}\right)$. O C $\mathrm{C}_{0}$ representa a variância não explicada, ou ao acaso, frequentemente, causada por erros de medições, ou variações dos
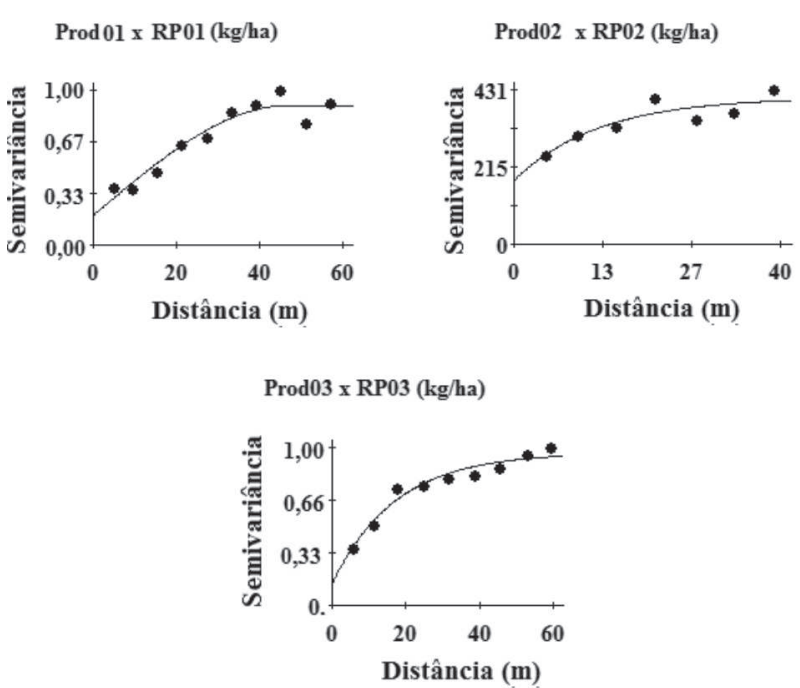

Figura 2: Semivariogramas cruzados da Produtividade (Prod) em função do número de ramos produtivos (RP) para três safras do cafeeiro conilon. 
atributos, que não podem ser detectados na escala de amostragem, e descreve o comportamento da função de correlação implícita no modelo, quando a distância entre amostras tende a zero.

Nos ajustes dos semivariogramas cruzados (Figura 2), verifica-se que o alcance para a Prod01xRP01 foi de $45 \mathrm{~m}$, próximo dos $42 \mathrm{~m}$ no semivariograma simples. Para a Prod02xRP02, o alcance aumentou 2,24 vezes, em relação ao alcance de $17 \mathrm{~m}$ e com aumento de 1,27 vezes para a Prod03xRP03. Esses resultados indicam que o método da cokrigagem estima, aproximadamente, valores em um raio de ação maior para a produtividade. Com isso, trabalha-se com o quantitativo de vizinhos maior que o da krigagem, na dependência espacial, com maior correlação espacial. Sendo assim, as informações relacionadas com os valores dos ramos produtivos minimiza a variação espacial da produtividade, proporcionando maior continuidade espacial. Segundo Gontijo et al. (2012), variáveis que apresentam maiores alcances de dependência espacial tendem a se apresentarem mais homogêneas, espacialmente, na área.

Após a realização da krigagem ordinária e da cokrigagem e comparando-se os resultados da validação cruza-
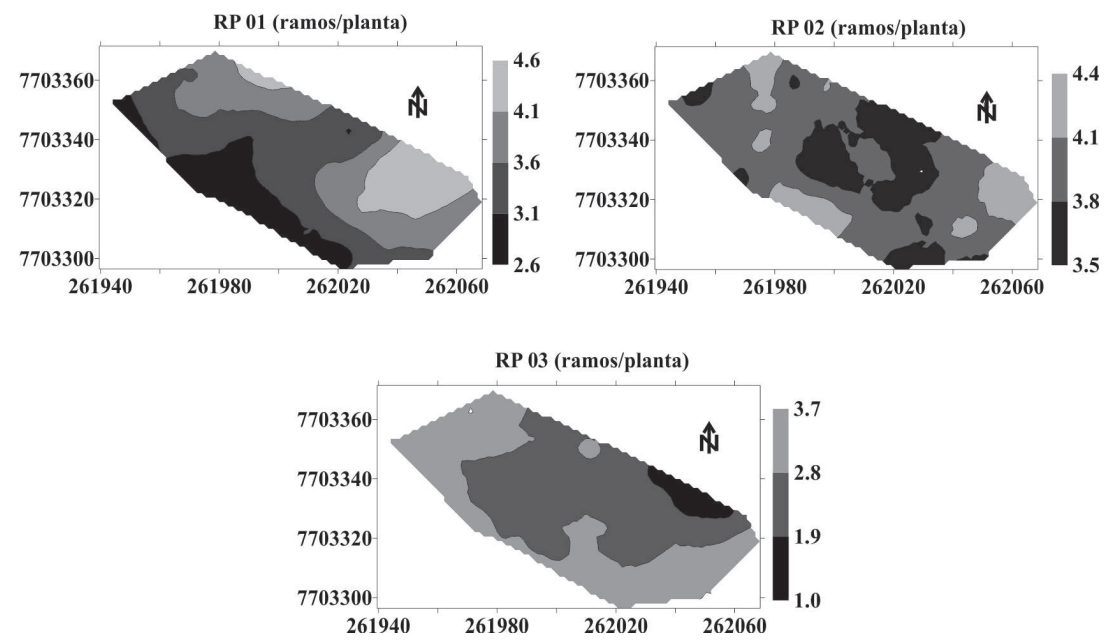

Figura 3: Mapas da distribuição espacial do número de ramos produtivos (RP) nas três safras de cafeeiro conilon, construídos por krigagem ordinária.
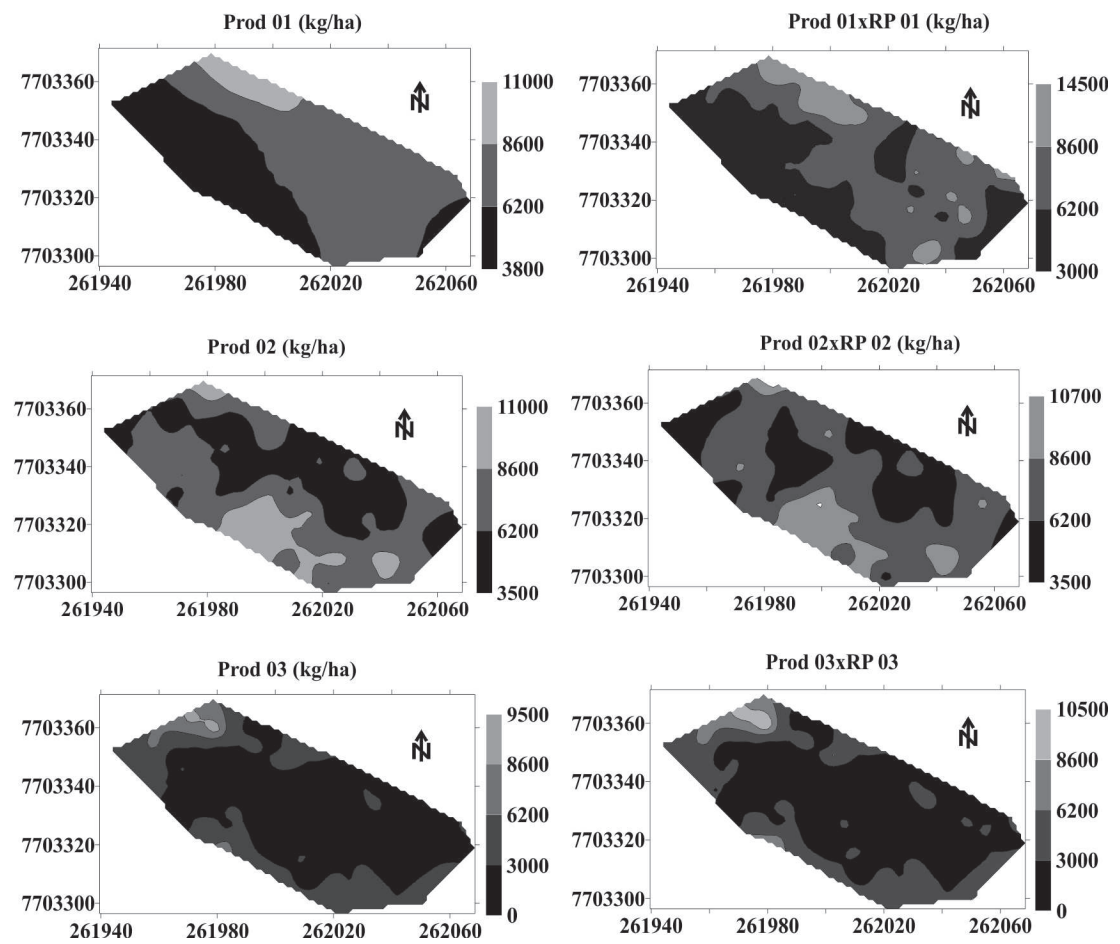

Figura 4: Mapas da distribuição espacial da produtividade em três safras, estimados pelo método krigagem ordinária (esquerda) e pela cokrigagem (direita). 
da, observam-se similaridades nos coeficientes de correlação (r-VC) (Tabela 3). Isso indica que a covariável ramo produtivo apresenta bons resultados na estimação da produtividade do cafeeiro conilon (Robusta Tropical), seco em coco.

Nas Figuras 3 e 4, estão apresentados os mapas da distribuição espacial das variáveis construídos por krigagem ordinária e cokrigagem, respectivamente.

Na primeira safra, o maior número de ramos produtivos (RP01) situou-se na parte central para a superior do mapa, que corresponde à parte de menores altitudes da área. Os RP02 apresentam-se em transição, com altos valores nas partes superior e inferior da Figura. Já a safra 03 apresentou maiores valores de ramos produtivos na parte baixa do mapa, que corresponde às maiores altitudes, ocorrendo uma inversão dos RP03 quanto à distribuição espacial, em comparação com a primeira safra.

Observa-se, quanto à forma, semelhança entre os mapas de produtividade, em cada safra, estimados pelos dois métodos (Figura 4). As pequenas diferenças são decorrentes do processo de estimação. Os valores médios das Prod01 e 02 (6636,5 e 6990,1 $\mathrm{kg} \mathrm{ha}^{-1}$ ) situam-se entre 6200 e $8600 \mathrm{~kg} \mathrm{ha}^{-1}$, maior região da área. A Prod03 apresenta os maiores valores acima do limite de $8600 \mathrm{~kg} \mathrm{ha}^{-1}$, em uma pequena região à direita do mapa, com baixa produtividade na região central da área, o que refletiu uma baixa produtividade média nessa safra.

Verifica-se, nos mapas das produtividades construídos por krigagem ordinária (esquerda), o mesmo padrão de distribuição espacial dessa variável, quando estimada pelos ramos produtivos, nas três safras, por cokrigagem (direita) (Figura 4), confirmando-se correlação espacial positiva entre as variáveis. Isso evidencia a importância de se considerar a dependência espacial, uma vez que essas observações não seriam possíveis, caso fossem considerados somente os valores médios das variáveis em estudo.

\section{CONCLUSÕES}

A produtividade e o número de ramos produtivos por ponto amostral apresentaram dependência espacial nas três safras.

A estimativa da produtividade pelo número de ramos produtivos apresenta similaridade na cokrigagem, indicando ser a covariável número de ramos produtivos (plagiotrópicos) eficiente na estimativa da produtividade.

\section{AGRADECIMENTOS}

Os autores agradecem ao CNPq, pela bolsa de produtividade do primeiro autor; à FAPES, pelos recursos financeiros para a realização deste trabalho e, ao INCAPER, pela cessão da área.

\section{REFERÊNCIAS}

Berlato MA, Farenzena H \& Fontana DC (2005) Associação entre El Nino Oscilação Sul e a produtividade do milho no Estado do Rio Grande do Sul. Pesquisa Agropecuária Brasileira, 39:423-432.

Bottega EL, Silva AS, Costa MM \& Bottega SP (2011) Cokrigagem na estimativa dos teores de $\mathrm{Ca}$ e $\mathrm{Mg}$ em um Latossolo Vermelho distroférrico. Revista Ciência Agronomica, 42:821-828.

Cambardella CA, Moorman TB, Novak JM, Parkin TB, Karlen DL, Turco RF \& Konopka AE (1994) Field-scale variability of soil properties in Central Iowa soils. Soil Science Society America Journal, 58:1501-1511.

Ferrão RG, Fonseca AFA, Bragança SM, Ferrão MAG \& De Muner LH (2007) Café Conilon. Vitória, Incaper. 702p.

Ferrão MAG, Ferrão RG, Fornazier MJ, Prezotti LC, Fonseca AFA, Alixandre FT, Costa H, Rocha AC, Moreli AP, Martins AG, Souza EMR, Araújo JBS, Ventura JA, Castro LLF \& Guarçoni RC (2008) Técnicas de produção de café arábica: renovação e revigoramento das lavouras no Estado do Espírito Santo. $1^{\mathrm{a}}$ ed. Vitória, Incaper, 56p.

Gontijo I, Nicole RN, Partelli FL, Bonomo R \& Santos EOJ (2012) Variabilidade e correlação espacial de Micronutrientes e matéria orgânica do solo com a produtividade da pimenta-do-reino. Revista Brasileira de Ciência do Solo, 36:1093-1102.

Goovaerts P (1997) Geostatistics for natural resources evaluation. New York, Oxford University Press. 483p.

Isaaks EH \& Srivastava RM (1989) Applied geostatistics: introduction to applied geostatistics. New York, Oxford University Press. 561p.

Journel AC \& Huijbregts CJ (1978) Mining geostatistics. Academic, London. 600p.

Kitamura AE, Carvalho MP \& Lima CGR (2007) Relação entre a variabilidade espacial das frações granulométricas do solo e a produtividade do feijoeiro sob plantio direto. Revista Brasileira de Ciência do Solo, 31:361-369.

Landim PMB, Sturaro JR \& Monteiro RC (2002) Exemplos de aplicação da cokrigagem. Rio Claro, UNESP. 19p. (Texto didático, 9).

Lima JSS, Oliveira RB \& Quartezani WZ (2007) Variabilidade espacial de atributos físicos de um solo sob cultivo de pimentado-reino. Engenharia na Agricultura, 15:291-298.

Lima JSS, Oliveira RB \& Silva AS (2009) Estudo da fertilidade do solo em lavoura de café conilon utilizando análise multivariada e geoestatística. In: Simpósio de geoestatística aplicada em ciências agrárias, Botucatu. Anais, FCA/UNESP. CD-ROM.

McBratney AB \& Webster R (1986) Choosing functions for semivariograms of soil properties and fitting them to sampling estimates. Journal of soil Science, 37:617-639.

Oliveira RB (2007) Mapeamento e correlação de atributos do solo e de plantas de café conilon para fins de agricultura de precisão. Dissertação de Mestrado. Universidade Federal do Espírito Santo, Alegre. $129 \mathrm{p}$.

Soares A (2006) Geoestatística para ciências da terra e do ambiente. Lisboa, IST Press. 214p.

Silva AS, Queiroz DM, Pinto FAC \& Santos NT (2014) Coffee quality and its relationship with Brix degree and colorimetric information of coffee cherries. Precision Agriculture, 15:543554.

Silva SA, Lima JSS, Souza GS, Oliveira RB \& Silva AF (2010a) Variabilidade espacial do fósforo e das frações granulométricas de um Latossolo Vermelho Amarelo. Revista Ciência Agronômica, 41:1-8. 
Silva SA, Lima JSS \& Souza GS (2010b) Estudo da fertilidade de um Latossolo Vermelho-Amarelo húmico sob cultivo de café arábica por meio de geoestatística. Revista Ceres, 57:560-567.

Silva SA, Lima JSS \& Alves AI (2010c) Spatial variability of the nutritional condition of canephora coffee aiming specific management. Bioscience Journal, 26:558-565.
Silva AF, Quartezani WZ, Zimback CRL \& Landim PMB (2011) Aplicação da geoestatística em ciências agrárias. Botucatu, FEPAF. 136p.

Warrick AW \& Nielsen DR (1980) Spatial variability of soil physical properties in the field. In: Hillel D (Ed.) Applications of soil physics. New York, Academic. p.319-344. 